\title{
Finite Element Analysis and Optimal Design of Portable Optical Fiber SPR food Detector based on SolidWorks
}

\author{
Zhaoming Chen \\ Chongqing Institute of Green and \\ Intelligent Technology, Chinese \\ Academy of Sciences. \\ Chongqing, P.R. China, 401122 \\ 023-63061481 \\ chenzhaoming@cigit.ac.cn
}

Shixuan He

Chongqing Institute of Green and Intelligent Technology, Chinese Academy of Sciences.

Chongqing, P.R. China, 401122

\author{
Yu Huang \\ Chongqing Institute of Green and \\ Intelligent Technology, Chinese \\ Academy of Sciences. \\ Chongqing, P.R. China, 401122 \\ 023-63061481 \\ huangyu@cigit.ac.cn \\ Lijuan Liu \\ Chongqing Institute of Green and \\ Intelligent Technology, Chinese \\ Academy of Sciences. \\ Chongqing, P.R. China, 401122
}

\author{
Jiming He \\ Chongqing Institute of Green and \\ Intelligent Technology, Chinese \\ Academy of Sciences. \\ Chongqing, P.R. China, 401122 \\ 023-63061481
}

\author{
Dongyun Tang \\ Chongqing Institute of Green and \\ Intelligent Technology, Chinese \\ Academy of Sciences. \\ Chongqing, P.R. China, 401122
}

\begin{abstract}
SolidWorks was used to create a three dimensional mechanical model of portable optical fiber SPR food detector. The total deformation and stress figures of the optical fiber SPR detector were analyzed by using SimulationXpress subjected to finite element analysis. In order to achieve the device's portability and stability, the framework of detector was optimized. The thicknesses of base plate, partition and safety coefficient were the variables for optimization. Benefitted from the compromise of design variables, the mass of device was reduced to be more reasonable while the mechanical quality of the detector had been maintained.
\end{abstract}

Keywords-Optimal design; Portable optical fiber SPR food detector; SolidWorks; Structural analysis;

\section{INTRODUCTION}

In recently years, optical fiber surface plasmon resonance (SPR) has attracted great interests due to its many applications ranging from environmental monitoring to analysis of biomaterials. Compared to the prism-based SPR sensor, the fiber-based SPR sensor has many advantages, such as simple in construction, cost efficiency and feasibility in remote sensing application. The usefulness of SPR in sensing device is due to its high sensitivity, precision and selectivity. Illnesses caused by pathogens and toxic food have serious impacts on the consumers' healthy. Therefore, it is necessary to inspect the food substances in the food production chain, from food producers, food processors to distributors and retailers. The portable optical fiber SPR detector takes advantages of fiber and sensitive SPR technology to meet the urgent demand of online inspection of food substances [1-8]. The portable optical fiber SPR detector is able to distinguish the categories of food substance. Then its launch could be used to minimize the negative effect of food contamination on the consumer's healthy and economic development. However, the mechanical vibration is a major factor to affect the quality of detected signal from the point of mechanical view. In order to obtain high quality signal with minimized mechanical vibration noise, SolidWorks 10.0 was used to design and analysis of portable optical fiber SPR detector's mechanical framework. The mechanical analysis and verification of the structure was implemented by SimulationXpress module of SolidWorks. An optimized framework was introduced to achieve the reliability and stability of portable optical fiber SPR detector.

\section{ESTABLISHMENT OF OPTICAL FIBER SPR FOOD DETECTOR MODEL}

Due to the rapid development of computer technology, finite element analysis algorithm is a powerful tool in the field of numerical simulation and mechanical analysis to design an equipment in order to improve design efficiency and save cost. SolidWorks makes use of finite element analysis method to design mechanical framework automatically.

In the modeling process of portable optical fiber SPR detector, bottom-up method was used to sketch the basic structure of various detectors' modules according to their shape and size, and assist stretching boss/matrix, rotating boss/matrix, stretching resection to create a solid model of the detector. It should maintain the independence of physical characteristics and ignore the characteristics such as screw thread, sharp corners and round, which had little effect on the model structure. More attention was paid to check every component in the structure to ensure there is no interference between each component. 
Portable optical fiber SPR food detector was composed of an optical fiber spectrometer, a halogen light source, a Y-shape coupled fiber and the SPR fiber sensor deposited with thin gold film. The dimension of portable optical fiber SPR food detector was $320 \mathrm{~mm}$ (Length) $\times 300 \mathrm{~mm}$ (Width) $\times 260 \mathrm{~mm}$ (Height). The thickness of base plate and partition were $4 \mathrm{~mm}$ and $3 \mathrm{~mm}$ respectively. The three dimensional solid model of the detector is shown in Fig.1.

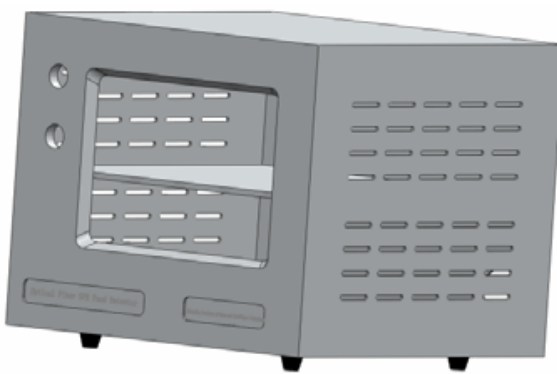

Figure 1. Solid model of optical fiber SPR food detector

\section{FinITE ELEMENT ANALYSIS}

The method of finite element analysis simulates mechanical status of equipment and calculates its corresponding mechanical response. The typical analysis process follow these steps: establishing three dimensional models, solving with loads and constraint, and analyzing computing results [9-12]. Its analysis flow chart is shown in the Fig.2. The gridding, applying loads and constraints were important steps to guarantee the computing accuracy and solving precision. The analysis of computing results was a validation and assessment step for analytical processing.

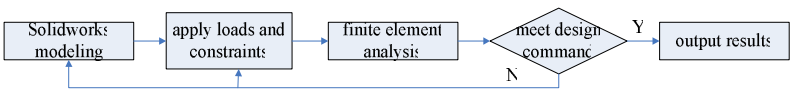

Figure 2. Flow chart of finite element analysis

\section{A. Finite Element Gridding}

The finite element gridding is a crucial step for the numerical simulation analysis, which directly affected the accuracy of subsequent numerical calculation results [13-15]. By increasing the number of chamfer, rounding, holes and surfaces, this model became more complex, which resulted in the difficulty of gridding and analysis of convergence. Therefore, the structure of detector was simplified to smoothly grid.

The size and shape of grids, as the main parameters of gridding, had a major impact on the calculation results. If gridding was too fine, more computer resource was needed to get good modeling at the expense of long time for calculation and the difficult connection of gridding between complex structures of different grids. Therefore, a trade-off between grid size and computer capability was necessary. The computer used in this modeling has four $2.83 \mathrm{GHz}$ CPUs and 3.4 GB memory. The automatic grid method could improve the efficiency of calculation. The total number of units by using automatic grid method in the modeling was 22929 and the total nodes number was 49516 after gridding. The gridding result of detector entity is shown in Fig.3.

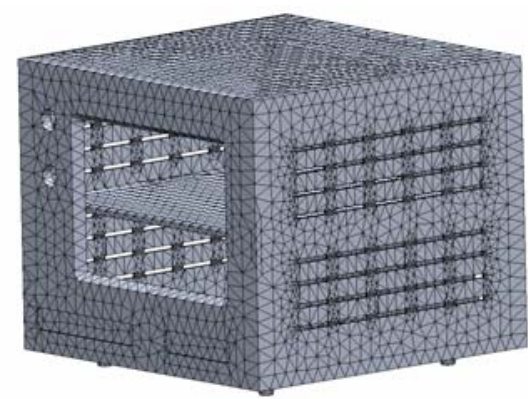

Figure 3. Finite element grid of the detector entity

\section{B. Loads and Constraints}

The loads and constraints fitted with the reality of engineering exercise had a direct impact on the accuracy and rationality of the analysis. In practical work, the maximum load and the maximum amplitude conditions were used to analyze the most adverse conditions. The shell material was set to be aluminum alloy. The supported seat material was set to be rubber. Solving parameters are showed in Table 1.

TABLE 1. PARAMETERS FOR CALCULATION

\begin{tabular}{lll}
\hline \multicolumn{1}{c}{ Materials } & Solving parameter & \multicolumn{1}{c}{ Value } \\
\hline \multirow{4}{*}{ Aluminum alloy } & Density $\rho$ & $2700\left(\mathrm{~kg} / \mathrm{m}^{3}\right)$ \\
& Elastic modulus E & $69(\mathrm{GPa})$ \\
& Yield strength $\sigma$ & $27.57(\mathrm{MPa})$ \\
& Poisson's ratio v & 0.33 \\
\hline \multirow{4}{*}{ Rubber } & Density $\rho$ & $1300\left(\mathrm{~kg} / \mathrm{m}^{3}\right)$ \\
& Elastic modulus E & $7.84(\mathrm{MPa})$ \\
& Yield strength $\sigma$ & $19.61(\mathrm{MPa})$ \\
\hline
\end{tabular}

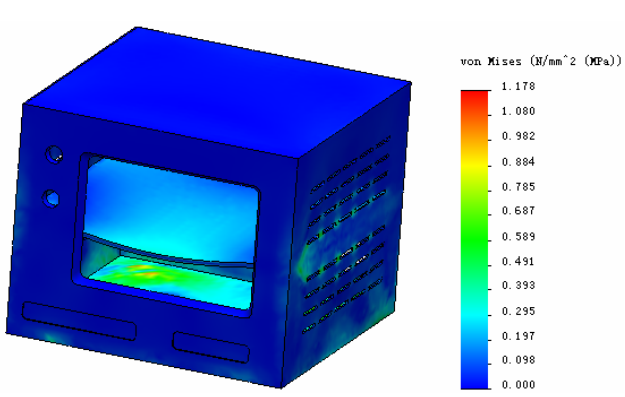

Figure 4. Stress nephogram of detector.

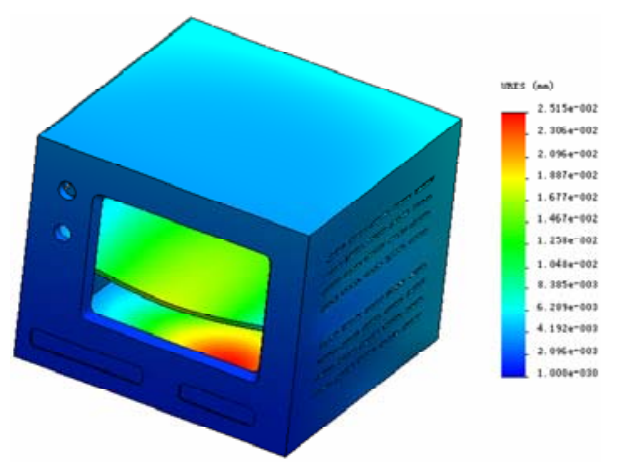

Figure 5. Displacement nephogram of detector 
Constraints of 4 supported seats on the bottom plate were fixed constraint, the vertical downward force applied on bottom plate, applied pressure on the base plate and partition respectively. The stress cloud chart and displacement cloud chart of this computing are shown in Figs. 4 and 5. The deformation ratio in the two figures was 1272 . Figure 4 shows the maximum stress of the detector applied on the base plate and conformed to the reality. The maximum stress value was $1.178 \mathrm{MPa}$. Compared to the yield strength, the minimum safety coefficient is $\mathrm{n}=27.57 / 1.178=23.404$. Figure 5 shows that the maximum displacement of $0.025 \mathrm{~mm}$ was extremely small. A large margin existed in the safety coefficient and displacement, which is waste in the design. It is necessary to optimize this framework in the strength and stiffness of the structure by reducing the structure mass and saving materials under the premise of safety and reliance.

\section{OPtimal Design of PORTABLE OpticAl Fiber SPR FOOD DETECTOR}

The simulation integrated optimization module SimulationXpress was used to optimize the structure. The design principle was to reduce the mass of detector as much as possible under the premise of strong strength, rigidity and stability. The bottom plate and partition had large stress and deformation parameters to modify in order to implement this optimization. The overall dimensional parameter of the detector was maintained in order to avoid significant change in the system's structure.

The optimized thickness of the base plate(x1) and the partition(x2) were set to be $2.5 \mathrm{~mm}$ and the optimized safety coefficient was set to be 10.0 respectively. It should be noted that default optimization range in the module SimulationXpress might cause error due to model geometric conditions. As a result, optimal value was obtained after 20 times of simulation. The data related to this optimization is shown in Table 2. The strength and rigidity of optimized model was re-finite element analysis and its strength and rigidity were improved. Its mass was decreased from $4.298 \mathrm{~kg}$ to $3.916 \mathrm{~kg}$, which indicated that the optimization process could reduce the structural mass and save materials.

\section{CONCLUSION}

SolidWorks was used to establish three-dimension model of the portable optical fiber SPR food detector. Simplification and optimization of this model was implemented by using SimulationXpress tools where the applied loads and constraints on this device were considered. The structure of detector was optimized in accordance with the requirements of stabilizing its framework. The reduction of its mass and improvement of safety coefficient were achieved by optimized the device' structure to maintain its stability and portability.

TABLE2. DATA BEFORE AND AFTER OPTIMIZATION

\begin{tabular}{|c|c|c|c|c|c|c|}
\hline \multicolumn{3}{|c|}{ Design variables } & \multicolumn{3}{|c|}{ Constraint condition } & \\
\hline $\mathrm{x} 1(\mathrm{~mm})$ & $\mathrm{x} 2(\mathrm{~mm})$ & $\begin{array}{c}\operatorname{maximum} \\
\text { stress(MPa) }\end{array}$ & & $\begin{array}{c}\text { maximum } \\
\text { displacement }(\mathrm{mm})\end{array}$ & $\begin{array}{c}\text { Mass of } \\
\text { detector }(\mathrm{kg})\end{array}$ & \\
\hline Original data & 4.0 & 3.0 & 1.178 & 0.025 & & 4.298 \\
\hline Optimized data & 3.2 & 2.5 & 2.740 & 0.073 & & 3.916 \\
\hline
\end{tabular}

\section{REFERENCES}

[1] Rajan, Subhash Chand, B.D. Gupta, "Surface Plasmon resonance based fiber-optic sensor for the detection of pesticide," Sensors and Actuators B, vol.123, pp. 661-666, 2007.

[2] Simona Scarano, Marco Mascini, Anthony P.F. Turner, Maria Minunni, "Surface plasmon resonance imaging for affinity-based biosensors," Biosensors and Bioelectronics, vol.25, pp.957-966, January 2010.

[3] Barbora Špačková, Marek Piliarika, Pavel Kvasnička, Christos Themistos, Muttukrishnan Rajarajan, JiříHomola , "Novel concept of multi-channel fiber optic surface plasmon resonance sensor," Sensors and ActuatorsB, vol. 139, pp. 199-203, May 2009.

[4] Yinquan Yuan, Liyun Ding, Zhenqiang Guo, "Numerical investigation for SPR-based optical fiber sensor," Sensors and Actuators B, vol. 157, pp. 240-245,2011.

[5] Rajneesh K. Verma and Banshi D. Gupta, "Surface plasmon resonance based fiber optic sensor for the IR region using a conducting metal oxide film," JOSA A, vol. 27, pp. 846-851, 2010.

[6] Anuj K. Sharma, Rajan Jha, and B. D. Gupta,"Fiber-Optic Sensors Based on Surface Plasmon Resonance," A Comprehensive Review, IEEE Sensors Journal, vol. 7, pp. 1118-1129, 2007.

[7] Yin-quanYuan, Zhen-qiangGuo and Li-yun Ding, "Influence of metal layer on the transmitted spectra of SPR-based optical fiber sensor," OPTOELECTRONICS LETTERS, vol. 6, pp. 346-349, 2010.

[8] Yu-Cheng Lin, Woo-Hu Tsai, Yu-Chia Tsao, and Jiu-Kai Tai, “An
Enhanced Optical Multimode Fiber Sensor Based on Surface Plasmon Resonance With Cascaded Structure," IEEE Photonics technology letters, vol. 20, pp. 1287-1289,2008.

[9] Godfrey Onwubolu, "Computer Aided Engineering Design With Solidworks," Imperial College Press London, UK, 2011.

[10] Ping Hai-Tao, Wu Zhi-Lin, Mo Gen-Lin, "Improvement Measures and Application of Parametric Design of Machine Parts Based on SolidWorks API," Coal Mine Machinery, vol. 32, pp. 235-238, Jan 2011.

[11] L. Ertl and P. Houška, "Design of Mass Spectrometer Control in NI LabVIEW and SolidWorks,"MECHATRONICS, Part II, pp.197-202,2012.

[12] Hong YanXing, Yan BinZhang, Wu YangHuang, "Design and Simulation of Drum Solid-State Fermentation Equipment Based on Solidworks," Advanced Materials Research, vol.476, pp.438-442, February 2012.

[13] F. Wei, F.R. Wang, "3D Parametric Design for Steel Head frame of Coal Mine Based on Solidworks," Key Engineering Materials, vol. 455, pp. 340-344, December 2010.

[14] Tang Xin, Deng Yuanchao, "Model Analysis of Solid Carbide Taps Based on ANSYS Workbench and Solidworks," Tool Engineering. Vol. 45, pp. 33-34, April 2011.

[15] Liu Jiao, Li Hong-Bin,Qian Lina,Ni Fu-Sheng,Xu, "Wei-Guang,Finite Element Analysis of a Dredger Cutterhead Based on SolidWorks and ANSYS ," Machine Design and Research, vol. 26, pp. 64-66, May 2010. 